\title{
Filigrane
}

Écoutes psychothérapiques

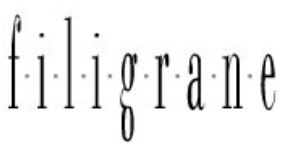

\section{Que reste-t-il de nos amours?}

\section{Catherine Chabert}

Volume 19, numéro 2, automne 2010

Adieu Oedipe, bonjour Narcisse ? II

URI : https://id.erudit.org/iderudit/1000454ar

DOI : https://doi.org/10.7202/1000454ar

Aller au sommaire du numéro

\section{Éditeur(s)}

Revue Santé mentale au Québec

\section{ISSN}

1192-1412 (imprimé)

1911-4656 (numérique)

Découvrir la revue

\section{Citer cet article}

Chabert, C. (2010). Que reste-t-il de nos amours ? Filigrane, 19(2), 9-20. https://doi.org/10.7202/1000454ar

\section{Résumé de l'article}

La tendance est forte aujourd'hui, qui consiste à considérer le complexe d'Oedipe comme caractéristique des névroses, et à penser d'autres modalités de fonctionnements psychiques en termes exclusivement préoedipiens.

Peut-on vraiment considérer que l'accès à l'oedipe soit réservé à certains et pas à d'autres ? Ou bien peut-on raisonnablement penser que, comme l'écrit Freud, " chaque nouvel arrivant dans le monde humain est mis en devoir " d'en venir à bout ? Dans cette perspective, ce sont les modes d'organisation et d'élaboration du complexe d'Oedipe, ses voies de résolution aussi, qui marqueront sa spécificité et ses différences : nous devrions alors admettre que les formes oedipiennes sont variables et singulières, qu'elles n'obéissent donc pas à un prototype. L'auteur propose, à partir de ce questionnement, un certain nombre de réflexions cliniques et métapsychologiques soutenant la nécessité de maintenir la référence au complexe d'Oedipe, et d'analyser ses articulations singulières avec le narcissisme et l'angoisse de perdre l'amour de la part de l'objet. Loin d'être exclusives, ces problématiques se conjuguent dans des configurations plurielles qui permettent de saisir les liens entre les différents registres de la psychosexualité.
Ce document est protégé par la loi sur le droit d'auteur. L’utilisation des services d'Érudit (y compris la reproduction) est assujettie à sa politique d'utilisation que vous pouvez consulter en ligne.

https://apropos.erudit.org/fr/usagers/politique-dutilisation/ 


\title{
Que reste-t-il de nos amours ? ${ }^{1}$
}

\author{
Catherine Chabert
}

\begin{abstract}
La tendance est forte aujourd'hui, qui consiste à considérer le complexe $\mathrm{d}^{\prime}$ CEdipe comme caractéristique des névroses, et à penser d'autres modalités de fonctionnements psychiques en termes exclusivement préœdipiens. Peut-on vraiment considérer que l'accès à l'œdipe soit réservé à certains et pas à d'autres? Ou bien peut-on raisonnablement penser que, comme l'écrit Freud, " chaque nouvel arrivant dans le monde humain est mis en devoir " d'en venir à bout? Dans cette perspective, ce sont les modes d'organisation et d'élaboration du complexe d'CEdipe, ses voies de résolution aussi, qui marqueront sa spécificité et ses différences: nous devrions alors admettre que les formes œdipiennes sont variables et singulières, qu'elles n'obéissent donc pas à un prototype. L'auteur propose, à partir de ce questionnement, un certain nombre de réflexions cliniques et métapsychologiques soutenant la nécessité de maintenir la référence au complexe $d^{\prime} \in \mathcal{E}$ dipe, et $d$ 'analyser ses articulations singulières avec le narcissisme et l'angoisse de perdre l'amour de la part de l'objet. Loin d'être exclusives, ces problématiques se conjuguent dans des configurations plurielles qui permettent de saisir les liens entre les différents registres de la psychosexualité.
\end{abstract}

1 y a, dans les nombreuses notes qui jalonnent les différentes éditions des Trois essais, quelques lignes consacrées, très brièvement, au complexe d'Edipe et à ses effets :

On dit à juste titre que le complexe d'Édipe est le complexe nucléaire des névroses et constitue l'élément essentiel de leur contenu. En lui culmine la sexualité infantile, laquelle influence de façon décisive la sexualité de l'adulte par ses effets ultérieurs. Chaque nouvel arrivant dans le monde humain est mis en devoir de venir à bout du complexe d'Edipe; celui qui n'y parvient pas est voué à la névrose. Le progrès du travail psychanalytique a souligné de façon toujours plus nette cette signification du complexe d'Edipe; la reconnaissance de son existence est devenue le schibboleth qui distingue les partisans de la psychanalyse de leurs adversaires. (1905, p. 170, note de 1920)

Rappelons d'abord que les Trois essais ont été entièrement rédigés dans la première édition, et même dans celle de 1910, sans référence explicite au complexe d'Edipe. Je veux dire sans que le terme même soit évoqué - même si la question des attractions incestueuses et la confrontation à leurs interdits est présente, même si, 
d'emblée, la sexualité humaine se construit dans un système biphasé, séparé par la pause ou la suspension de la latence. Que l'ensemble de l'ouvrage donc, mais aussi que toute l'œuvre de Freud jusqu'en 1910 se développe hors de l'œdipe peut nous laisser rêveurs. Et pourtant, nous sommes, à certains égards, confrontés à cette absence ou à cette négligence.

Une sexualité infantile sans œdipe est-elle pensable? Pourrions-nous envisager la construction de la névrose infantile, son après-coup, sa reviviscence dans l'analyse - et dans la vie - sans que les différentes composantes de ce conflit, fondamental, fondateur même, soient prises en compte?

La tendance est forte aujourd'hui, qui consiste à considérer le complexe d'Edipe comme caractéristique des névroses, et à penser d'autres modalités de fonctionnements psychiques en termes exclusivement préœedipiens. Peut-on vraiment considérer que l'accès à l'œdipe soit réservé à certains et pas à d'autres? Ou bien peut-on raisonnablement penser que, comme l'écrit Freud, «chaque nouvel arrivant dans le monde humain est mis en devoir » d'en venir à bout? Dans cette perspective, ce sont les modes d'organisation et d'élaboration du complexe d'CEdipe, ses voies de résolution aussi, qui marqueront sa spécificité et ses différences: nous devrions alors admettre que les formes œdipiennes sont variables et singulières, qu'elles n'obéissent donc pas à un prototype.

Si la clinique psychanalytique a vu s'élargir les limites de ses indications, c'est aussi au nom de cette ouverture que certains abandonnent, dans ces cas, la référence à l'œdipe en considérant que l'essentiel de la maladie psychique relève de graves difficultés dans les capacités d'élaboration de l'angoisse de perte, auxquelles les obstacles narcissiques sont généralement associés. Ce constat n'a pas à être démenti. La question devrait être formulée le plus clairement possible si l'on veut en analyser les développements et les avatars : à parler de narcissisme et d'angoisse dépressive, doiton pour autant oublier l'appartenance de l'un et de l'autre au champ de la psychosexualité? Devons-nous établir une ligne de démarcation effective entre ce qui relève des investissements narcissiques et ce qui engage les investissements objectaux? $\mathrm{Ne}$ peut-on penser - métapsychologiquement mais d'abord cliniquement - une dialectique qui, pour chacun, suit des mouvements contradictoires, mais aussi complémentaires? À partir du moment où ces interrogations utilisent le mot - galvaudé lui aussi, il est vrai - d'investissements, elles nous entraînent immanquablement dans le champ pulsionnel et, dans ce champ là, la sexualité occupe sa place, quelle que soit la théorie à laquelle on s'attache : celle qui dessine l'opposition entre pulsions d'autoconservation et pulsions sexuelles; celle, combien discutée, qui désigne le double mouvement des pulsions de vie et des pulsions de mort. Devons-nous oublier que le narcissisme est un destin pulsionnel et que, de ce fait, il s'inscrit définitivement dans le domaine du sexuel, quel que soit son objet, même si parfois sa négativité l'entraîne aux limites du vivant?

La clinique psychanalytique est là pour insister et consolider ce point de vue. Elle montre, en effet, les liaisons étroites entre la sexualité et la perte, entre le renouvellement du complexe d'Edipe et la reviviscence de l'angoisse de perdre l'amour de 
la part de l'objet; il arrive que la solidarité de ces deux problématiques soit si forte quelle bascule dans la condensation. Comment alors continuer à penser, en les isolant, en les excluant presque mutuellement, ce qui relève du "pré»-œedipien et ce qui se révèle, discrètement ou bruyamment d'un œdipe flamboyant?

On a coutume de considérer deux grands modèles dans l'œuvre de Freud: le premier, celui de l'hystérie, représente les temps forts de la sexualité infantile et de la découverte de l'inconscient. Porté par les fantasmes de séduction, il nous entraîne du côté des mouvements pulsionnels et du refoulement, il inscrit la différence des sexes et des générations. On peut penser qu'il culmine dans l'émergence du complexe d'CEdipe et l'analyse de l'ambivalence, sans compter la force de son action dans l'invention et les développements de la méthode. Il condense l'espoir dans la résolution des symptômes et la confiance dans les constructions métapsychologiques.

Le second, le modèle narcissique, qui n'efface pas l'autre, trouve ses prémisses dès 1914-1915, à partir de Pour introduire le narcissisme et Deuil et mélancolie. Il porte les marques de la déception, de la reconnaissance du masochisme, du refus de guérir. Et pourtant, il est lourd de nouvelles inventions, d'avancées prémonitoires, d'un entêtement fructueux.

Ces deux paradigmes, à mon avis, continuent d'être pertinents : ils ne sont certes pas exclusifs et sont susceptibles d'être conjugués ensemble grâce à la grammaire compliquée qui les articule ou les sépare. C'est à partir des deux motifs qui peuvent en offrir des formes vivantes que je souhaite engager ma démarche dans la recherche des liaisons entre le complexe d'CEdipe et l'angoisse de perdre l'amour, motifs essentiels autour desquels les mouvements pulsionnels, la dynamique des fantasmes et les identifications scellent le devenir de la psychosexualité.

Rebecca a 25 ans, elle n'est plus une adolescente, mais elle ne se considère pas non plus comme une femme adulte. Elle demeure psychiquement arrêtée à ses 13 ans, comme si, en effet, une déchirure était venue couper le fil du temps. C'est une grande jeune fille, mince et délicate, qui semble s'efforcer de cacher tout ce qui pourrait constituer l'indice le plus minime de la séduction. Un visage émacié, une coiffure sévère, de grosses lunettes masquant la beauté de ses yeux et une apparence androgyne, voilà les premiers souvenirs que je garde d'elle. Depuis sa puberté mais aussi depuis la séparation de ses parents, elle passe des heures à scruter dans son miroir les signes les plus imperceptibles des imperfections de sa peau. Elle ne supporte pas la moindre aspérité, elle traque millimètre par millimètre tout ce qui, au regard ou au toucher, pourrait altérer un visage qui doit rester totalement lisse et sans défaut. Doisje préciser que ces signes ont toujours été invisibles pour moi et que, en dépit de ma myopie, je crois qu'en effet, ils étaient très peu apparents, sans doute grâce à l'art de 
les camoufler auquel Rebecca excelle. Chaque matin, chaque soir, elle part à la chasse de ces marques. Elle les attaque, elle veut les détruire, mais le résultat est là, chaque jour plus désespérant à ses yeux: non seulement ces signes se régénèrent, mais son acharnement compulsif à les faire disparaître laisse des traces qu'elle tente d'effacer à tout prix. Ses consultations en dermatologie et en endocrinologie se sont multipliées sans résultats. Et pour cause... ce qui commence à l'inquiéter et motive sa venue est un nouveau symptôme apparu il y a un an ou deux. Elle passe beaucoup de temps, chaque matin, de plus en plus de temps, à organiser ce qu'elle appelle son "planning». Il faudrait que chaque instant de sa journée entre clairement dans une case horaire définie. Le temps pris à la fabrication de son emploi du temps augmente sans cesse, ce qui l'oblige à recommencer indéfiniment pour tenir compte des retards accumulés. Même scénario, même rituel que pour sa peau. «Ma peau, mon temps, dit-elle, c'est l'enfer de ma vie. » Elle ne pense qu'à ça. Sauf que, quand même, elle s'inquiète de la misère de sa vie amoureuse et s'affole de son avenir professionnel.

C'est au hasard d'une énième consultation de dermatologie que l'idée lui est venue d'aller voir une psychanalyste. Sans conviction et sans espoir, ajoute-t-elle aussitôt, et pourtant la voilà qui parle, volubile, avide de dire, déversant un flot de paroles et de larmes comme si, tout à coup, une simple présence attentive faisait sauter les verrous de sa prison.

Pendant de longs mois, Rebecca s'active et s'efforce de me montrer que personne, ni elle bien sûr, ni moi évidemment, ne parviendra à la dégager de ses compulsions. Pour elle, aucun changement ne peut advenir. Elle proclame sans cesse son indépendance et la force de ses déclarations est à la mesure de sa position interne qui, de mon point de vue, maintient une dépendance extrême vis-à-vis de ses objets, dépendance dont le soubassement essentiel relève d'une répétition aliénante qui soumet le moi à l'emprise de l'objet et du narcissisme. Dans cette perspective, on peut considérer que beaucoup de conduites compulsives témoignent de la dépendance au symptôme lui-même, qui s'instaure inéluctablement, s'entretient et s'emballe parce qu'il sert de masque et de représentant de l'objet. Le fondement de la compulsion relève de la lutte paradoxale contre la passivité, au sens où j'ai proposé de la définir à partir de Freud, c'est-à-dire comme "l'être excité par l'autre», mais un autre qui peut se prendre au filet de la relation spéculaire où l'un est l'autre et l'autre est l'un. Ce que je cherche à dire, dans cette brève présentation initiale de la cure de Rebecca, c'est que nous sommes bien là confrontés à une forme d'impasse narcissique, butée maligne qui marque de son sceau l'engagement transférentiel.

La version de son histoire, par Rebecca, était péremptoire. Elle en soulignait avec l'humour et la causticité qui la caractérisaient, le caractère banal, presque vulgaire, si commun disait-elle, qu'il en devenait lui-même blessant, car il signait une représentation d'elle-même qu'elle défendait coûte que coûte: elle était quelconque, affirmait-elle. Elle était moyenne, médiocre et sans intérêt, alors qu'elle aurait souhaité par-dessus tout être quelqu'un.

Une histoire banale donc: ses parents ne s'étaient jamais aimés ou plutôt non, son père n'avait jamais aimé sa mère. Il avait accepté de l'épouser à cause d'elle, 
Rebecca, une enfant dans le dos! Le couple avait mal tenu pendant treize ans - Rebecca évacuait littéralement la naissance des deux garçons venus après elle - puis avait décidé de se séparer. La mère en était toujours meurtrie, sa haine contre son mari n'avait jamais désarmé et s'aggravait du fait de sa solitude. Le père, quant à lui, était un monstre d'égoïsme et de cruauté inconsciente et chaque fois qu'elle parlait de lui, la voix de Rebecca montait de plusieurs tons. Pas de mots assez forts pour dire son mépris pour lui, la honte d'être sa fille, l'horreur qu'elle éprouvait chaque fois qu'il s'approchait d'elle. Et pourtant, elle était la seule de toute la famille à continuer à le rencontrer. Bref, la figure du père, objet de toute sa rage et de son affliction, occupait une grande place sur la scène de son théâtre intérieur. Et je lui dis un jour qu'elle avait le même acharnement à l'accabler afin de le faire disparaître de ses pensées que celui avec lequel elle torturait son visage. Je pensais, ce faisant, que c'était là une véritable entreprise de défiguration qui visait, en effet, davantage le père (et sans doute la mère) mais dont elle se déclarait la première victime.

La mère, en contraste, provoquait chez elle une soumission et une obéissance alarmantes. Rebecca guettait chez elle - elle la scrutait, elle aussi - le moindre signe de tristesse, de désintérêt, d'ennui ou d'irritation, et la perception des états d'humeur de sa mère ordonnait ses propres mouvements de plaisir ou de déplaisir. Pas question de la quitter, d'aller vivre un peu plus loin d'elle. Ce serait répéter l'abandon du père, sa fuite odieuse, sa lâcheté et son égocentrisme détestables. Elle se devait de rester là, toujours, indéfectiblement affectée à son poste: une sentinelle, une gardienne du temple, une vestale?

Je pouvais littéralement lui appliquer le propos de Freud dans Les métamorphoses de la puberté, lorsqu'il invoque la tâche qui incombe aux adolescents - s'affranchir de leur dépendance vis-à-vis des figures parentales, et la difficulté de certains (et surtout de certaines!) à l'accomplir :

En même temps que ces fantasmes manifestement incestueux sont surmontés et rejetés, s'accomplit une des réalisations psychiques les plus importantes, mais aussi les plus douloureuses de la période pubertaire: l'affranchissement de l'autorité parentale, grâce auquel est créé l'affranchissement entre la nouvelle et l'ancienne génération, si important pour le progrès culturel [...]. Il y a des personnes qui n’ont jamais surmonté l'autorité des parents et ne leur ont pas retiré la tendresse qu'ils leur vouaient, sinon de manière très imparfaite. Il s'agit pour la plupart de filles qui, à la joie de leurs parents, persistent bien au-delà de la puberté dans un amour filial absolu [...]. On apprend par là que l'amour apparemment non sexuel pour les parents et l'amour sexuel s'alimentent aux même sources, ce qui revient à dire que le premier ne correspond qu'à une fixation infantile de la libido. (1905, p. 171)

J'aurais pu, en effet, entièrement interpréter la répartition des investissements de Rebecca dans la perspective d'une fixation narcissique à la mère, aux effets d'une confrontation à une figure maternelle déprimée, défaillante, insuffisante à satisfaire les besoins d'étayage de son enfant. Evidemment, je ne néglige pas cet aspect dont je 
pense qu'il est, de toute façon, régulièrement présent et actif: ne pas le reconnaître serait contraire à la conception psychanalytique de la construction des identifications et, là-dessus, je reviendrai un peu plus tard.

Mais je cherche à souligner que l'exclusion du tiers, du père, de la compréhension théorique et clinique de telles situations relève, à mon avis, d'un piège transférentiel : celui qui pousserait Rebecca (et d'autres bien sûr) à démontrer qu'à l'instar de son attachement indéfectible et totalitaire à sa mère, personne, en dehors de son analyste - et encore fallait-il que le déplacement ait eu lieu - ne comptait désormais pour elle.

Rebecca avait passionnément aimé un garçon de son âge rencontré en Terminale. Passionnément, sans jamais lui montrer le plus petit signe d'attraction, voire en évitant systématiquement tout risque d'intimité entre eux, alors qu'elle était convaincue de la réciprocité de leur attirance. Elle considérait que le jeune homme était aussi timide et idéaliste qu'elle, elle se berçait d'attentes et de rêves jusqu'au jour où finalement il tomba dans les bras d'une autre. C'est très précisément à ce moment là que les programmations d'emploi du temps se mirent frénétiquement en place. La douleur de cette déception restait intacte. Depuis, Rebecca partageait sa vie amoureuse entre de brèves liaisons avec des hommes qu'elle méprisait et de longues aventures virtuelles sur Internet.

À cet égard, on pourrait tout à fait se représenter la vie amoureuse de Rebecca sur le modèle symétrique d'Un type particulier de choix d'objet chez l'homme développé par Freud en 1910, dans lequel apparaît pour la première fois le «complexe d'Edipe». Le rabaissement des hommes nourrissait une bonne part des propos de Rebecca : ils étaient indélicats, brutaux, mais aussi plutôt niais, grossiers, peu intelligents et elle prenait un vif plaisir à ces portraits qu'elle dessinait dans une langue intransigeante, incisive, acérée, mêlant toujours la rage et les larmes dans une sorte de plainte infinie infiltrée de revendications blessantes et sans cesse entretenues. En même temps, elle maintenait enfouie au plus profond d'elle-même, comme un trésor jalousement gardé, une figure de père à la fois idéale et excitante, à laquelle elle restait fondamentalement attachée.

Avec moi, l'enjeu était double: dans ses difficultés à admettre une liaison féconde entre nous deux, elle préservait et son lien à sa mère et son lien à son père puisque, justement, son analyse ne lui permettait en aucune manière de rencontrer un autre homme. Le problème était que, à son avis, dans la réalité, son père et sa mère, tout en étant séparés, restaient effectivement seuls et que, dans cette situation, Rebecca pouvait se penser comme l'objet d'amour privilégié de l'un et de l'autre, et donc coupable de les avoir séparés, d'autant plus coupable qu'inconsciemment elle réalisait pleinement ses désirs œdipiens; même si, bien entendu, cette représentation était cachée derrière une autre, inversée et portée par les reproches qu'elle se faisait de les avoir contraints à se marier du fait de sa conception.

C'est au cours de cette période, bien difficile, où elle réagissait très négativement à ses réussites - elle venait d'être reçue major de son école et avait été très « remarquée» pour la qualité exceptionnelle de ses travaux - qu'elle vint un jour, complète- 
ment bouleversée. Jusqu'ici, elle s'était toujours plainte de la distance de son père, de son manque d'intérêt pour ses enfants, de son avarice, de son caractère obsessionnel... bref, je l'ai déjà évoqué, ce père n'avait aucune grâce à ses yeux. Elle arrive donc ce jour là, fébrile et tempétueuse: elle vient de découvrir une photo que son père avait faite d'elle, enfant (elle devait avoir quatre ou cinq ans). Sur la photo, elle est debout sur une table, et "prise par en-dessous». Tout s'organisait désormais dans sa tête: son père était un pervers, un voyeur et il avait, pour sa fille, une attraction incestueuse. C'était la cause, disait-elle, de tous ses malheurs à elle. Elle avait dû être traumatisée et sans doute qu'elle continuait d'en subir les effets. Sa haine pour son père était évidemment exacerbée par cette trouvaille, mais en même temps, le sens qu'elle donnait à la photo justifiait en quelque sorte après coup l'état d'extrême excitation dans lequel toute évocation de ce père la plongeait. Dans les mois qui suivirent, elle s'acharna de plus belle contre son visage et s'engouffra dans un mouvement d'automortification inquiétant. En même temps, elle déplorait l'échec de son analyse tout en développant une féroce jalousie à l'égard des autres, ces patients qui avaient l'air d'être si heureux de vivre qu'elle me soupçonnait de leur consacrer tous mes soins, le meilleur de moi-même alors qu'elle se sentait négligée : les autres étaient, avec évidence, beaucoup plus intéressantes. "IntéressantES », relevai-je, à tout hasard ? Oui, elle pensait que les autres femmes étaient beaucoup plus intéressantes qu'elles. Elle en voulait pour preuve, l'intérêt de son père pour les autres femmes justement, la séduction qu'il semblait exercer sur elles et la rage dans laquelle cela la plongeait: comment un homme aussi peu agréable, aussi déplaisant pouvait déployer un tel charme? Les ruminations incessantes qui la tourmentaient à ce sujet étaient une torture: elle ne comprenait pas pourquoi elle s'acharnait à ce point contre elle-même alors que le coupable, c'était lui, ce père indigne, et sans valeur qu'elle vouait aux gémonies et dont elle souhaitait violemment être débarrassée.

Revenons un instant vers quelques considérations métapsychologiques ayant trait notamment aux identifications d'une part, au surmoi d'autre part, les deux étant d'ailleurs liés. Pour ce faire, je me référerai à des textes postérieurs aux Trois essais, en remarquant cependant que les différentes éditions "augmentées» correspondent chacune à des découvertes "nouvelles", à des remaniements importants dans le cours de l'œuvre de Freud: 1910, 1915, 1920, 1924. Cela nous rappelle en effet que le complexe d'Edipe a été présenté en 1910, que Pulsions et destins des pulsions paraît en 1915 - entre Pour introduire le narcissisme et Deuil et mélancolie - que Au-delà du principe de plaisir paraît en 1920 et que en 1923, Le moi et le ça est édité. Ce que je veux dire par là est que nous ne saurions faire un bond de 1905 à 2005 sans prendre en compte les modifications apportées par Freud aux Trois essais, modifications largement déterminées par l'évolution de son expérience et de sa pensée.

Plus précisément, je voudrais m'attarder sur Le moi et le ça, et notamment sur deux chapitres de cet essai - «Le moi et le surmoi» et «Les états de dépendance du 
moi» - parce qu'ils me paraissent particulièrement éclairants pour la recherche de points de liaison entre odipe et angoisse de perte d'amour de la part de l'objet. Parce qu'ils traitent des identifications dont nous savons qu'elles sont héritières du complexe d'CEdipe, et inéluctablement associées à l'abandon de l'objet. Évidemment, et déjà par rapport à Rebecca, le parallélisme développé par Freud entre névrose obsessionnelle et mélancolie m'intéresse. Non que je veuille m'engager dans une discussion diagnostique, mais parce que chez la jeune femme, j'ai pu repérer des traits psychiques qui appartiennent à ces deux affections, et surtout parce que les fins rouages qui articulent les deux modes de fonctionnement sont justement analysés dans la double référence aux identifications et à l'édification du surmoi, entendus comme résultant des voies d'élaboration de la perte. Cette comparaison entre névrose obsessionnelle et mélancolie met en évidence avec éclat comment deux affections psychiques qui, au niveau de leur organisation respective, relèvent d'entités psychopathologiques très différentes s'inscrivent cependant dans un continuum: le parallélisme entre les deux montre aussi de quelle manière l'ancrage et les effets du surmoi sont susceptibles de trouver des formes plurielles tantôt liées par un tressage libidinal qui en assure la vitalité, tantôt emportées par un déchaînement (au sens littéral) pulsionnel destructeur.

Aux commencements, explique Freud, il est bien difficile de distinguer investissement d'objet et identification. Le moi, encore faible, ou bien consent aux tendances érotiques et aux investissements d'objet, ou s'en défend (on voit déjà se dessiner deux destins pulsionnels possibles). Si le moi abandonne l'objet sexuel ou y est contraint, il se passe, comme dans la mélancolie, une intériorisation de l'objet perdu. Comment se développe cette substitution? Par une régression à la phase orale? Par une introjection qui rend possible l'abandon de l'objet? En tout cas, et cette assertion est essentielle, le caractère du moi résulte de la sédimentation des investissements d'objets abandonnés, il contient l'histoire de ces choix d'objet. C'est là, il me semble, que les propositions de 1923 sont vraiment décisives pour saisir la continuité des expériences psychiques.

Notons d'ores et déjà à quel point, pour Freud, la condensation des expériences précoces et de celles qui suivent le cours de la vie est massive. À quel point cette condensation nécessite, pour être analysée, que se défassent les liaisons trop serrées entre le passé précoce et la suite des événements psychiques. À quel point la décondensation peut nous fourvoyer parfois dans la recherche d'une chronologie établie qui, bien entendu, semble clarifier la complexité des phénomènes psychiques, mais peut tout autant entraîner des dérives notoires non seulement dans la compréhension des conduites psychiques, mais aussi et surtout dans les modalités d'usage de la méthode analytique.

La clinique actuelle ne doit pas nous faire oublier cette consubstantialité de la perte et du sexuel. Certes, il ne s'agit pas de nous détourner de la détresse au sens le plus freudien du terme, mais de l'entendre comme une partition à plusieurs voix dont on ne saurait se défaire de la pluralité. Il est sûr, en effet, que les voies de traitement et d'élaboration de la perte et les identifications qui en découlent obéissent à 
des procédures différentes selon que le choix d'objet s'établit de manière prévalente sur un mode objectal ou narcissique. Les prémisses de ce mouvement sont déjà très fermement établies dans la comparaison entre deuil et mélancolie mais aussi dans la mise en perspective des identifications hystériques et narcissiques. Pourtant, à l'époque, cette claire opposition a essentiellement une valeur didactique et psychopathologique. C'est plus tard, en 1923, que Freud s'inspire très directement de la mélancolie pour suivre le déroulement «naturel» des processus identificatoires au cours du développement.

Pour Freud, je le rappelle, une des instances considérées comme la plus différenciée dans la seconde topique, le surmoi, tient ses sources de deux facteurs biologiques essentiels : le long état de détresse et de dépendance infantile du petit homme, et le fait de son complexe d'CEdipe pris dans l'instauration de sa vie sexuelle. Evidemment, en nous approchant de cette haute instance dont nous savons à quel point les formes et les effets sont variables, c'est bien pour revenir à ses origines dans la rencontre entre la détresse et CEdipe. Le surmoi a une position particulière dans le moi, parce qu'il est la première identification qui se soit produite alors que le moi était faible et parce qu'il est l'héritier du complexe d'Edipe et a donc introduit dans le moi des objets de la plus grande importance.

Pensons simplement à l'impact évident des difficultés d'élaboration de l'angoisse de perdre l'amour de la part de l'objet sur la résolution du complexe d'Edipe. Celleci exige le renoncement aux objets d'amour originaires, et sera donc plus ou moins lourdement entravée selon la manière dont ce renoncement est éprouvé: une perte douloureuse, irrémédiable ou un abandon impossible à accepter. Déjà dans cette procédure, le renoncement œedipien qui constitue l'une des composantes majeures du complexe et les modalités de traitement de la perte entrent en résonance flagrante dans l'amorce et le déroulement du déclin de l'œedipe. Les qualités de son héritier - le surmoi - seront tributaires de la force de ses racines pulsionnelles mais sans doute aussi de leurs objets.

Freud décrit au sujet du surmoi deux positions presque paradoxales: «Le surmoi conserve tout au long de la vie le caractère que lui a conféré son origine dans le complexe paternel, c'est-à-dire la capacité de s'opposer au moi et de le maîtriser ». Et d'autre part: «Il est le mémorial de la faiblesse et de la dépendance qui étaient jadis celles du moi et il perpétue sa domination même sur le moi maturé. »

Nous retrouvons là l'écho persistant de Deuil et mélancolie: dans la mélancolie, la libido libérée par l'abandon de l'objet, au lieu de s'attacher à un nouvel objet, est ramenée vers le moi et sert «à instaurer une identification du moi avec l'objet abandonné». L'ombre de l'objet tombe sur le moi, qui est alors «jugé», le conflit entre le moi et la personne aimée se transforme en scission entre la critique du moi et le moi modifié par l'identification. En même temps, un double mouvement caractérise le travail mélancolique : bien sûr, le moi se déprécie et fait rage contre lui-même et, en ce sens, sa «faiblesse» demeure. Mais conjointement, en soubassement, l'objet lui aussi est dévalorisé, rabaissé, abattu. Le moi alors peut jouir de la satisfaction à se reconnaître légitimement comme supérieur à l'objet. 
Ce mouvement peut être repérable dans certains destins œedipiens. Le déplacement des investissements sur un nouvel objet ne se réalise pas du fait de leur faiblesse libidinale: la déception entraîne un abandon de l'objet aimé, un désinvestissement à la mesure de l'attaque narcissique qu'elle implique. C'est dans cette situation que l'ombre d'CEdipe peut tomber sur le moi. Nous pouvons, en même temps, nous approcher un peu plus de la notion d'objet interne: celui-ci n'est pas simplement la représentation introjectée d'une figure de la réalité ou même d'une image, il est plutôt constitué par le réseau compliqué des mouvements pulsionnels, des identifications et des fantasmes qu'il condense, il est davantage une configuration plutôt qu'une incarnation.

Chez Rebecca, coexistent deux objets d'attaque incessante, son père et elle, pris ensemble à la fois dans une spécularité scellée par des identifications narcissiques évidentes et en même temps dans une conflictualité œdipienne non moins agissante. Dans cette bataille acharnée, la mère reste en apparence préservée, sauf que, entre Rebecca et elle, le réseau narcissique et œdipien est tout autant mobilisé. Par ses états d'humeur dépressive, par sa solitude et son refus du plaisir, Rebecca colle à l'image de sa mère qu'elle entretient avec passion: en affichant la permanence de son insatisfaction, elle cherche à montrer, à endosser même, l'absence de toute satisfaction chez sa mère. La dynamique narcissique et son aboutissement dans le reflet et le miroir conduisent à une affirmation en double sous-tendue par un déni d'importance: le désir de la fille est abrogé en union avec le refus de perception de celui de la mère. Cependant, cette dynamique narcissique ne constitue qu'un versant de la problématique. L'autre se décline, avec évidence, en termes œdipiens: nier le désir entre la mère et le père revient à dénier le lien sexuel entre eux, en maintenant l'impossibilité infantile de se représenter la sexualité des parents, en réalisant aussi le souhait de séparation du couple, en se rebellant contre une exclusion menaçante imposée par leur vie amoureuse.

Les efforts incessants de Rebecca pour exclure toute représentation de la scène primitive, le refus d'en admettre l'incidence et donc la force du refoulement qui en assurait l'éloignement, se révèlent à la mesure de ses manifestations les plus étrangères. Ce qui surgit chez elle avec une violence bouleversante pourrait pourtant bien être associé au fantasme: la scène de la photo, scène de séduction s'il en est, où elle n'entend pas ses propres mots - «prise par en-dessous »- relève d'un léger déplacement, en apparence, mais d'un déplacement lourd de conséquences. Dans la scène de séduction, au-delà de ses accusations contre la "perversité» de son père, elle occupe une place centrale, condensant le triomphe de l'enfance et de la féminité.

Et dans le même temps, c'est à son analyste qu'elle se montre, dévoilant les « dessous » de ses plaintes, et du même coup les annulant puisque c'est comme «bienaimée » qu'elle se présente à moi désormais. Bien-aimée du père parce qu'elle est sa seule fille, parce qu'elle lui ressemble dans sa combativité et la vitalité de son goût de 
vivre; bien-aimée parce que, finalement, leur amour mutuel a surmonté l'épreuve de la séparation. Bien-aimée qui doit aussi payer pour ces péchés, pour le plaisir si fort qu'il a dû être effacé, caché au dehors, refoulé au dedans, pour ce plaisir infantile qui doit rester secret.

Etre aimé du surmoi, même au minimum, est la condition pour pouvoir rester vivant car le surmoi assure, par identification et intériorisation, un regard bienveillant pour le moi, à l'instar de celui du père qui interdit parce qu'il aime et protège. Mais il est aussi une formation du moi issue du complexe d'Edipe ou au moins consolidée par lui : il relève, comme toute identification, d'une sédimentation à partir de laquelle un double mouvement s'accorde - identification au père et à la mère dans le cadre d'un œedipe complet. Double identification dans la constitution même du surmoi : l'une qui s'ancre sur l'état d'impuissance et de faiblesse du moi infantile, en quête de protection; l'autre, arrimée au développement de l'œdipe, réclamant, elle aussi, un interdit préservant de la confusion incestuelle. Ce que, déjà en 1905, Freud appelait «La barrière de l'inceste» reste la condition pour que la part prise à l'autre, l'appropriation du trait par identification, maintienne la dimension vivante de l' «être comme », compromis effectif entre la frustration et la satisfaction du désir - être comme, certes, mais ailleurs, hors du champ originaire du fantasme.

Ainsi, la capacité à renoncer sans être détruit peut être considérée comme un produit de la fonction protectrice du surmoi, de son effectivité. Ce destin de la culpabilité met en évidence la part vivante du moi, son empreinte libidinale, indéfectiblement attachée à l'objet alors que l'orientation mélancolique, elle, l'abandonne et s'engouffre dans la voie narcissique vite enlisée par le parasitage d'un objet introjecté certes mais désanimé, détruit, mort.

En redécouvrant et en reconnaissant son amour pour son père (à la mesure de sa haine pour lui), Rebecca en éprouve bien sûr la dimension transgressive, presque criminelle. Mais en même temps, l'amour du père pour elle constitue un formidable étayage, une fois désamorcées les convictions incestueuses: c'est un père à la fois tendre et exigeant, préoccupé de sa fille, étonnamment présent dans la réalité de sa vie, qui se dessine peu à peu. C'est là que le surmoi tyrannique et sévère auquel Rebecca s'était masochistement soumise trouve une autre source, bienveillante, qui l'autorise enfin à abandonner ses compulsions auto-destructrices.

Freud a insisté sur les corrélations inconscientes entre l'attachement au père et à la mère, en soulignant notamment que, derrière tout grand amour pour le père se cache - et se découvre - un grand amour pour la mère. Peut-on poser la même question à propos de la haine?

Au début du traitement de Rebecca, j'ai été très vite convaincue du renversement en son contraire de son immense amour pour son père. Son hostilité déclarée, sa fougue, ses rougeurs, ses larmes, allaient vraiment dans le sens d'une extrême ambivalence dont le complexe d'EEdipe proposait une orchestration partielle certes, 
mais qui n'en permettait pas moins une distribution pulsionnelle sur la mère et le père. C'est cette partition qui se déploie dans le transfert et en permet l'analyse. Je ne crois pas qu'il eût été possible d'aborder et surtout d'interpréter d'emblée l'ambivalence de Rebecca vis-à-vis de sa mère. C'est forte de l'analyse de son lien à son père - dont, bien sûr, sa mère n'était pas absente -, forte aussi de son ambivalence à mon égard, qu'elle put vraiment s'y confronter, et d'abord, en admettant enfin que sa mère avait été l'objet du désir de son père. À cette mère-là, à cette jeune mère de l'enfance, les voies de l'identification étaient ouvertes et avec elles une possible résolution de la névrose infantile.

\section{Catherine Chabert \\ 76, rue Charlot, 75003 Paris \\ catherine@chabert.org}

\section{Note}

1. Ce texte, partiellement remanié, a fait l'objet d'une conférence à la Fédération européenne de psychanalyse en mars 2008.

\section{Références}

FREUD, S., 1905, Les métamorphoses de la puberté, in Trois essais sur la théorie sexuelle, Gallimard, Paris, 1987, 141-175.

FREUD, S., 1910, Un type particulier de choix d'objet chez l'homme, in La vie sexuelle, PUF, Paris, 1969, 47-55.

FREUD, S., 1914, Pour introduire le narcissisme, in La vie sexuelle, PUF, Paris, 1969, 263-324.

FREUD, S., 1915, Pulsion et destins des pulsions, in Métapsychologie, Gallimard, Paris, 1971, 11-44.

FREUD, S., 1917, Deuil et mélancolie, in Métapsychologie, Gallimard, Paris, 1971, 147-174.

FREUD, S., 1920, Au-delà du principe de plaisir, in Essais de psychanalyse, Payot, Paris, 1981, 41-115.

FREUD, S., 1923, Le moi et le ça, in Essais de psychanalyse, Payot, Paris, 1981, 219-275. 